\title{
MPR et blessure de guerre
}

\section{Physical medicine and rehabilitation and war-related injuries}

\author{
É. Lapeyre \\ (C) Springer-Verlag France 2013
}

De la blessure à la réparation, tel est l'enjeu pour le service de santé des Armées et ses services de médecine physique et de réadaptation mis en exergue ces dernières années par le conflit en Afghanistan, qui aura fait 87 morts et plus de 700 blessés français.

Conflit asymétrique, il a été marqué par l'utilisation de nouveaux agents vulnérants, en particulier les engins explosifs improvisés (IED) responsables de lésions de « blast», dont nous vous décrirons la physiopathologie et la présentation clinique.

Le développement des protections individuelles du combattant et les progrès de la médecine de « l'avant » ont amélioré le pronostic vital de manière significative tout en augmentant le nombre de blessés graves aux lésions multiples et complexes.

Au-delà du temps médical horizontal multidisciplinaire hospitalier et des phases initiales de rééducation, afin de mener à bien les projets de réadaptation et de réinsertion de ces blessés, il a fallu pour les services de MPR développer une action verticale en pleine synergie avec la hiérarchie militaire via les Cellules d'Aide aux Blessés.

C'est ainsi qu'a été créée au sein de l'hôpital d'Instruction des Armées (HIA) Percy la C2RBO (cellule de réadaptation et réinsertion des blessés en opération). Cette entité expérimentale permet à la fois de proposer un projet de réadaptation et de réinsertion personnalisé à chaque combattant blessé, mais également de constituer une force de proposition pour le commandement, afin que soit améliorée la prise en charge globale de ces blessés.

Pour illustrer ce propos, nous vous détaillerons le comparatif de la prise en charge des blessés de guerre américains et français, et plus spécifiquement l'approche française multidisciplinaire et intégrative constituant, à notre sens, un modèle assez original, depuis la phase initiale jusqu'à la phase de réadaptation et de réinsertion. La finalité est d'être, lorsque cela est possible, « au plus près du régiment, au plus près de la fonction ». Les premiers résultats de cette réinsertion

\section{É. Lapeyre $(\bowtie)$}

Professeur agrégé du Val-de-Grâce,

chef du service de médecine physique et réadaptation,

hôpital d'instruction des Armées de Percy,

101 avenue Henri Barbusse, F-92141 Clamart cedex

e-mail : ericlapeyre@hotmail.com sociale et professionnelle à travers la cohorte suivie à l'HIA Percy jusqu'en 2010 seront analysés.

Nous avons aussi choisi, de manière didactique, de vous montrer à travers l'exemple de la prise en charge de l'amputation traumatique de guerre, les problématiques auxquelles nous sommes confrontés à la fois dans le diagnostic et la prise en charge thérapeutique de ces blessés.

Ainsi l'entité clinique des « pieds de pont», véritables « pieds de blast » illustre pour nous toute la difficulté que nous avons, dans un cadre multidisciplinaire, à décider avec le patient d'une stratégie conservatrice ou d'une amputation secondaire.

Une fois l'amputation réalisée, nous avons souvent été confrontés, probablement en raison des lésions tissulaires liées au blast, à la survenue d'ossifications hétérotopiques.

Notre préoccupation a été d'emblée de faire bénéficier notre population jeune, active et sportive d'amputés de membre des matériels prothétiques les plus performants. L'arrivée sur le marché des prothèses bioniques de nouvelle génération (mains et genoux) a été pour nous un enjeu tout à la fois médicotechnique (essayer de valider leur pertinence) et médico-économique (obtenir le remboursement de ces dispositifs). Nous venons de l'obtenir pour nos blessés en opération. Ceci ouvre pour nous un champ actif de recherche en appareillage (plasticité cérébrale, évaluation biomécanique, échelle fonctionnelle spécifique, etc.)

En matière de rééducation neurologique, notre attention se focalise actuellement sur les conséquences du blast sur l'encéphale. Nous mettons en place un projet de recherche qui vise à analyser plus finement le lien entre le traumatisme crânien léger (mild TBI des Anglosaxons) et l'état de stress post-traumatique. Il est en effet indéniable que les lésions physiques liées au blast et les lésions psychiques sont très souvent associées.

Tout ceci témoigne, vous le voyez mes chers collègues, de la dynamique qui s'est installée au sein des services de MPR des Armées. Ils sont parfaitement intégrés dans le cadre d'actions multidisciplinaires à la prise en charge du combattant blessé.

En espérant que ce numéro spécial des équipes « militaires ", coordonné avec l'aide de mon collègue David Rogez, puisse constituer un apport supplémentaire au dynamisme de notre spécialité.

Bonne lecture. 